\title{
Filtered Carrier Phase Estimator for High-Order QAM Optical Systems
}

\author{
Rozental, Valery; Kong, Deming; Corcoran, Bill; Mello, Darli A.A.; Lowery, Arthur J.
}

Published in:

Journal of Lightwave Technology

Link to article, DOI:

$10.1109 / J L T .2018 .2829153$

Publication date:

2018

Document Version

Early version, also known as pre-print

Link back to DTU Orbit

Citation (APA):

Rozental, V., Kong, D., Corcoran, B., Mello, D. A. A., \& Lowery, A. J. (2018). Filtered Carrier Phase Estimator for High-Order QAM Optical Systems. Journal of Lightwave Technology, 36(14), 2980-2993.

https://doi.org/10.1109/JLT.2018.2829153

\section{General rights}

Copyright and moral rights for the publications made accessible in the public portal are retained by the authors and/or other copyright owners and it is a condition of accessing publications that users recognise and abide by the legal requirements associated with these rights.

- Users may download and print one copy of any publication from the public portal for the purpose of private study or research.

- You may not further distribute the material or use it for any profit-making activity or commercial gain

- You may freely distribute the URL identifying the publication in the public portal

If you believe that this document breaches copyright please contact us providing details, and we will remove access to the work immediately and investigate your claim. 


\title{
Filtered Carrier Phase Estimator for High-Order QAM Optical Systems
}

\author{
Valery Rozental, Deming Kong, Member, IEEE, Bill Corcoran, Member, IEEE, Darli Mello, Member, IEEE, and \\ Arthur James Lowery, Fellow, IEEE
}

\begin{abstract}
We investigate, using Monte Carlo simulations, the performance characteristics and limits of a low-complexity filtered carrier phase estimator (F-CPE) in terms of cycleslip occurrences and signal to noise ratio (SNR) penalties. In this work, the F-CPE algorithm has been extended to include modulation formats whose outer-ring symbols have a QPSK symmetry, and which are applicable to metro and long-haul optical networks: QPSK, 8, 16, and 64QAM. The proposed jointpolarization approach, where the number of non-null symbols in a simplified QPSK partition is increased, shows a further improvement in robustness against cycle slips, resulting in cycleslip-free operation at symbol rate $32 \mathrm{GBd}$ and laser linewidths up to $900 \mathrm{kHz}$, for the range of investigated SNRs. In addition, it reduces SNR penalties for only a small incremental complexity. We also propose a method for constellation alignment that exploits F-CPE computational blocks to minimize the electronic footprint, in order to compensate for an arbitrary rotation, introduced by F-CPE. We further show that F-CPE can successfully recover the phase of a 32QAM signal that does not have the outer-ring QPSK symmetry, albeit with increased penalties and higher cycleslip rates. A performance comparison between F-CPE, the blind phase search algorithm, and a data-aided phase estimator, is also presented.
\end{abstract}

Index Terms-Optical communications, coherent optical systems, digital signal processing, carrier recovery, cycle-slips.

\section{INTRODUCTION}

The ever growing demand for increased transmission rates in optical systems requires the use of spectrally efficient highorder modulation formats beyond QPSK, where the associated transmission distance limitations are partially overcome by the high gain of soft-decision forward error correction (FEC) codes. High-order modulation formats are extremely sensitive to phase noise, caused by the non-null spectral width of transmitter and local oscillator lasers.

Digital signal processing blocks in coherent receivers commonly compensate for the phase noise using all-feedforward, highly parallelized architectures. Feedforward is used because symbol rates are tens of times higher than the internal ASIC clock frequency, which in turn creates a processing delay for feedback loops [1]. In QPSK-based 100G systems, and in a general $m$-PSK case, phase noise can be efficiently compensated using the feedforward Viterbi \& Viterbi algorithm [2], which uses the rotational symmetry of $m$-PSK constellations

Valery Rozental, Bill Corcoran, and Arthur Lowery are with the ElectroPhotonics Lab, Department of Electrical \& Computer Systems Engineering, Monash University, Clayton, Australia (e-mail: valery.rozental@monash.edu) Deming Kong is with the High-Speed Optical Communications group, Technical University of Denmark (DTU), Lyngby, Denmark. Darli Mello is with the School of Electrical and Computer Engineering, State University of Campinas (UNICAMP) - SP, Brazil. to map onto a single point of the I-Q plane when raised to the $m$-th power-an operation known as information removal. Nevertheless, higher-order QAM constellations, which have better additive noise tolerance than PSK, do not possess the information removal property. One way to tackle this problem is through QPSK partition, where high-order QAM constellations are divided into QPSK clusters [3], [4], [5], [6], [7]. The QPSK partition increases computational complexity, especially when the, so called, non-class-one symbols are rotated, or otherwise transformed (e.g., [4], [5], [7]). Another approach, known as the blind phase search (BPS) [8], is based on best "fitting" of the $m$-QAM constellation into different rotation angles. In addition to good performance characteristics, BPS can be efficiently implemented in hardware using a high degree of parallelization. A significant drawback of BPS is its elevated computational complexity, especially for a high-order QAM. Additional works have built on the idea of BPS, aiming to reduce its complexity and improve performance characteristics [9], [10], [11].

Another phase-noise compensation related issue is cycle slips_phase discontinuities of multiples of $\pi / 2$. Cycle slips are induced by the phase unwrapping operation [12], especially under low signal to noise ratios (SNRs), which is commonly the case for modern soft-decision FEC schemes. Differential decoding can be used to cope with cycle slips at the expense of sensitivity. Although sensitivity penalties associated with differential decoding decrease with a higher QAM order, differential decoding requires increased receiver complexity when implemented jointly with soft-decision FEC schemes [13]. Pilot-aided solutions, which aim to eliminate cycle slips, result in reduced spectral efficiency, and present additional challenges when the signal quality is poor [14]. Some cycleslip-tolerant FEC schemes that aim to reduce the associated computational complexity and penalties have been proposed (e.g., [13], [15], [16]). From the above discussion it follows that carrier recovery methods that completely avoid or greatly reduce the probability of cycle-slip occurrences are highly desirable. It is worth noting that BPS can in principle be made very robust against cycle slips by increasing the duration of the noise removal window, though this increases BPS computational complexity even further.

In [17] we introduced and experimentally validated a blind phase recovery algorithm based on tracking the low-frequency components of the phase noise, which we called the filtered carrier-phase estimator (F-CPE). The F-CPE performs suboptimal phase noise estimation, while aggressively rejecting additive noise. This approach makes F-CPE robust against 
cycle slips, and allows low-complexity implementation using frequency-domain filtering. F-CPE did not present cycle slips in 15- and 32-GBd 16QAM transmission experiment with external cavity lasers (ECL) with $\leq 100 \mathrm{kHz}$ linewidth, and could outperform BPS in terms of bit error rate (BER), for signal qualities comparable with FEC codes.

In this paper we extend our analysis, and offer the following contributions. Firstly, we apply the algorithm to additional modulation formats whose outer-ring symbols have a QPSK symmetry; that is, QPSK, 8QAM, 16QAM, and 64QAM. In addition, we show that F-CPE can be also used with 32QAM, whose outer-ring symbols do not have a QPSK symmetry; however, at the expense of a lower receiver sensitivity and reduced cycle-slip robustness. Secondly, we conduct extensive numerical analyses to establish performance characteristics, cycle-slip-free operation range and limitations, which can be used as design guidelines for ASIC implementation. We propose an architecture for joint-polarization phase recovery with limited incremental complexity. We further propose a method that performs I-Q alignment of the received constellation during system start-up. The associated architecture exploits existing DSP blocks to minimize the overall electronic footprint. Finally, we present a comparison between F-CPE, BPS, and a data-aided phase estimator, in terms of bit error rates, phase noise resilience, and cycle-slip occurrences.

The reminder of this paper is structured as follows. Section II reviews the F-CPE algorithm. A new joint-polarization architecture, and the constellation alignment method are introduced. Section III presents the numerical analysis, and Section IV presents our conclusions.

\section{Filtered CARrier Phase Estimator}

The block diagram of F-CPE is depicted in Fig. 1. It receives at its input a one sample-per-symbol equalized $m$ QAM constellation, impaired by additive noise and phase noise. Throughout this work we assume that any frequency offset between the carrier laser and the local oscillator has been previously compensated. Thus, the I-Q plane plot of the input constellations consists of concentric rings, whose number varies according to the modulation order: 1, 2, 3 and 9 rings for 4, 8, 16 and 64QAM, respectively. F-CPE comprises a QPSK partition block, raising to the fourth power, frequency-domain filtering, argument extraction, division of the argument by 4 to counteract phase-noise multiplication by the fourth-power operation, and phase unwrapping. In essence, the F-CPE we propose is a modified Viterbi \& Viterbi algorithm, whose novelties are a threshold-based, low-complexity, noise-minimizing QPSK partition, and an aggressive low-pass filtering, implemented in the frequency domain for higher computational efficiency, described hereafter.

The proposed QPSK partition strategy is based on selecting only the outer-ring symbols that form a QPSK constellation, shown in red in Fig. 2. The rationale behind this choice is illustrated in Fig. 3, which shows a first quadrant of an IQ plane for a 16QAM constellation. All constellation points lie on three concentric rings, R1-R3, of radii $r_{1}=\sqrt{E_{s} / 5}$, $r_{2}=\sqrt{E_{s}}$, and $r_{3}=\sqrt{9 E_{s} / 5}$, where $E_{s}$ is the average

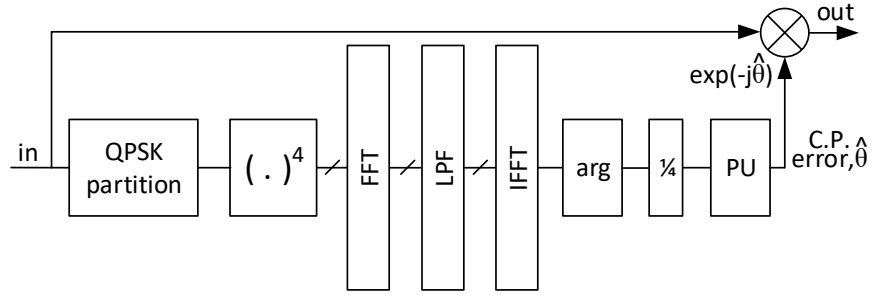

Fig. 1. Filtered CPE block diagram. (I)FFT, (inverse) fast Fourier transform; LPF, low-pass filter; PU, phase unwrapper; C.P., carrier phase.

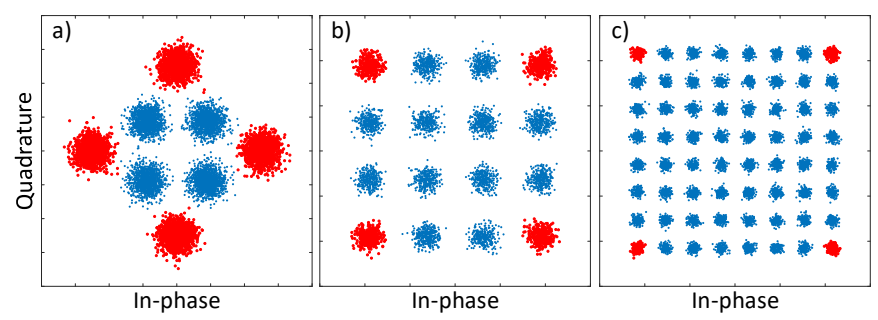

Fig. 2. QPSK partition by outer-ring symbols selection (in red) for (a) 8QAM; (b) 16QAM; and (c) 64QAM.

symbol energy. We wish to compare the accuracy of phase noise estimation for the three individual rings. Assume that a constellation point from each ring is corrupted by an identical sample, $\Delta \theta_{k}$, of the phase noise process, and identical sample, $n_{k}$, of a circularly symmetric additive Gaussian noise process (solid green). The phase noise sample causes a rotation by $\Delta \theta_{k}$, forming arcs along the rings (solid purple), whose lengths, $a_{1-3, k}=r_{1-3} \cdot \Delta \theta_{k}$, are proportional to the radii. Conversely, the arcs $n_{1-3, k}^{\prime}$, formed by projecting the additive

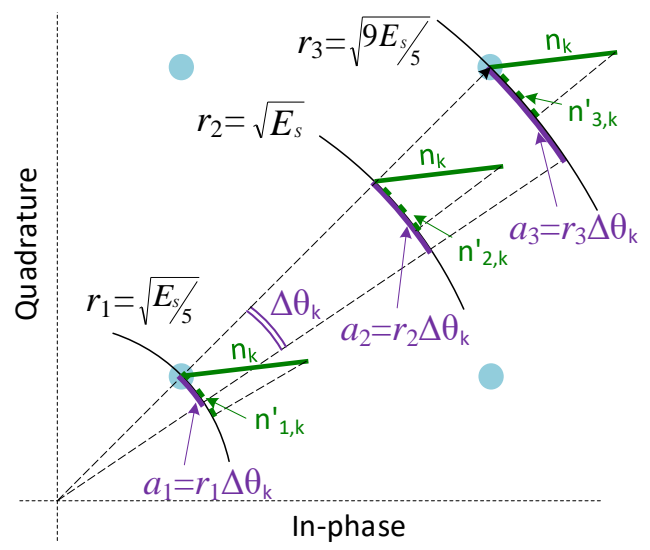

Fig. 3. Noise rejection through QPSK partition. Phase-noise-induced arcs, $a_{1-3}$ are proportional to the radii $r_{1-3}$, while additive noise projections, $n_{1-3, k}^{\prime}$, are similar in length; so that phase noise to additive noise ratio, $a / n^{\prime}$, is the highest for the outer ring, R3.

noise onto the three rings (dashed green) are similar in length (small differences due to the curvature variations are ignored here for simplicity). The phase noise estimation resumes in estimating $a_{1-3, k}$, impaired by $n_{1-3, k}^{\prime}$.

Conveniently, 16QAM symbols on R2 have the same energy as the constellation average, and can be used as a reference. Thus, for the outer-ring, R3, the phase-noise-induced arc, $a_{3}$, is $\sqrt{9 / 5}$ times longer than the average. Therefore, for carrier 
recovery, using only the outer-ring symbols is equivalent to having a $(\sqrt{9 / 5})^{2} \approx 2.55$-dB improvement in signal to noise ratio (SNR). Conversely, using only the inner-ring (R1) symbols is equivalent to a $(\sqrt{1 / 5})^{2} \approx 7$-dB SNR degradation. The same analysis shows that the equivalent SNR improvement from using only the outer-ring symbols for 8 and 64QAM is about 2 and $3.7 \mathrm{~dB}$, respectively.

The outer-ring symbols are detected by comparison with a threshold value. Symbols that lie below the threshold are set to zero. An additional advantage of such threshold-based partition is its simplicity, when compared to decision-directed schemes with subsequent de-rotation (cf. [5], [6]). Naturally, using only a limited subset of the constellation symbols for phase estimation will filter-out high-frequency phase-noise components, causing some performance penalties, quantified hereafter. [18]:

After QPSK partition, phase estimation can be described by

$$
\Delta \theta_{k}=P U_{4}\left\{\frac{1}{4} \arg \left[\sum_{n=-N / 2}^{N / 2} w_{n} x_{k+n}^{4}\right]\right\},
$$

where $w_{n}$ are the low-pass filter coefficients, $x_{k}$ are the resulting symbols after QPSK partition, $N$ is the FIR filter length, and $P U_{M}$ is the phase unwrapper operation, which constrains the incremental phase variation to the interval $[-\pi / M, \pi / M]$ by adding multiples of $\pm 2 \pi / M$ whenever absolute phase variation between consecutive elements is greater than $\pi / M$. One possible implementation of phase unwrapper is as follows [12], [19]:

$$
P U_{M}\{.\}=\{.\}+\left(\left\lfloor\frac{1}{2}+\frac{\Delta \theta_{k-1}-\{\cdot\}}{2 \pi / M}\right\rfloor\right) \frac{2 \pi}{M} .
$$

It has been shown that optimal phase estimation filter in a minimum mean-square error sense consists of pre- and post-cursor symmetric exponentially decaying sequences, dependent on the ratio between the phase noise and additive noise [12]; though, without taking into account the threshold position for QPSK partition. Conversely, F-CPE offers a suboptimal solution, compromising for aggressive additive noise rejection through QPSK partition and narrow bandwidth lowpass filtering (in the order of tens of megahertz). This aggressive filtering makes F-CPE robust against cycle-slips, albeit with some performance penalties. Fortunately, these penalties become significant only for high signal qualities, higher than required for modern hard- and soft-decision FEC schemes [17]. Further, low-pass filtering of the QPSK-partitioned symbols can be efficiently implemented in the frequency domain using the fast Fourier transform (FFT) algorithm, which may make F-CPE attractive from the computational complexity perspective, particularly when compared to BPS and its variants.

Averaging of the additive noise through joint-polarization processing has been extensively used in carrier recovery [3], [19], [20], [21]. In this work, we also investigate jointpolarization processing, whose architecture is shown in Fig. 4. Here, we assume that the inter-polarization phase difference has been previously compensated. The proposed architecture adds only a small incremental complexity, because filtering and subsequent stages remain identical to the baseline architecture of Fig. 1. There is an additional threshold-based QPSK partition and a raising to the fourth power. This structure is similar to the flat-filter feedforward carrier recovery architecture proposed in [19]. However, in [19], the sum of the QPSK-partitioned symbols of the two polarizations is further divided by two (an averaging operation). Conversely, FCPE does not require averaging because after QPSK partition the probability of outer-ring symbol occurrence in a single polarization is much higher than the probability of outerring symbol occurrence in both polarizations simultaneously. For example, ignoring the threshold influence and admitting error-free detection of outer-ring symbols, the probability of simultaneous occurrence of outer-ring symbols, R3, in both polarizations for $16 \mathrm{QAM}$ is $1 / 4 \times 1 / 4=1 / 16$, while the probability of occurrence of outer-ring symbols in a single polarization is $1 / 4 \times 3 / 4+1 / 4 \times 3 / 4=6 / 16$.

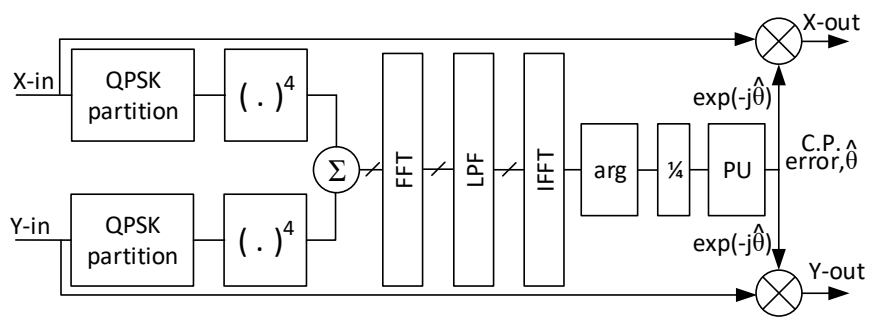

Fig. 4. Joint-polarization processing in F-CPE.

Throughout the experimental validation in [17], it was observed that F-CPE can produce an arbitrarily misaligned constellation. This misalignment can be removed using the architecture shown in Fig. 5. Here, the diagram shows the baseline architecture of Fig. 1 with additional blocks, required for I-Q alignment, highlighted in blue. The alignment mechanism consists of a parallel path, where the non-null symbols after QPSK partition are raised to the fourth power, corrected by $\exp \{-j 4 \hat{\theta}\}$, and stored in a buffer. When the buffer is full, an average deviation of the argument from $\pi$ is computed. The key motivation here is to maximize the use of existing blocks, so that incremental ASIC footprint is minimized. This alignment operation does not contribute significantly to the computational complexity, because it only has to run in the background in a much larger time-frame than symbol rate (e.g., performed after every 10 million symbols). One drawback of this architecture is that it requires a pointer mechanism for storing the positions of the non-null symbols, so that

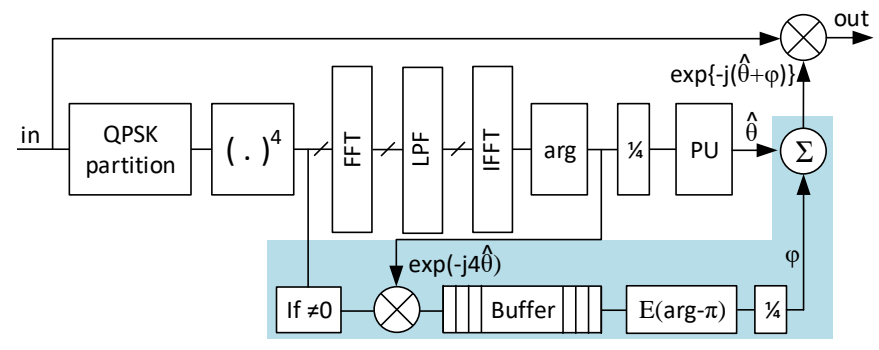

Fig. 5. Proposed architecture for system start-up I-Q alignment. 
their phase correction occurs in the corresponding instants. Alternatively, the condition block (if $\neq 0$ ) can be dropped, and all symbols stored in buffer indiscriminately. In this way, only the fixed filtering processing latency is considered, at the expense of a much larger buffer size.

\section{NUmERICAL ANALYSIS}

\section{A. Numerical model and algorithms settings}

Our numerical model, implemented in MATLAB ${ }^{\mathrm{TM}}$, uses additive white Gaussian noise (AWGN) to emulate the amplified spontaneous emission (ASE) of the erbium-doped fiber amplifiers in long-haul optical links, and a discretetime Wiener process to emulate phase noise. The Wiener process has incremental step $\Delta \theta=\theta_{k+1}-\theta_{k}$ that is normally distributed: $\Delta \theta \sim \mathcal{N}\left(0,2 \pi \Delta \nu T_{s}\right)$, where $\Delta \nu$ is the sum of carrier and local oscillator laser linewidths, and $T_{s}$ is the symbol interval [12]. All variables in our analysis are set with respect to a 32-GBd symbol rate signal.

Following the findings in [17], throughout this paper we use a Hamming-window-designed FIR low-pass filter (LPF) of order 200 (filter order $=$ number of taps -1 ). In particular, in [17] it was found that increasing the filter order above 200 produces only marginal sensitivity improvement. The Hamming window is defined in the discrete-time domain as [22]:

$$
w_{H}[n]=\alpha-\beta \cos (2 \pi n / N)
$$

where $\alpha=0.54 ; \beta=0.46$, and $N$ is the number of non-null samples (equal to the number of filter taps). Its discrete-time Fourier transform (DTFT) is given by [23]:

$$
W_{H}(f)=\alpha W_{R}(f)+\frac{\beta}{2} W_{R}\left(f-\frac{f_{s}}{N}\right)+\frac{\beta}{2} W_{R}\left(f+\frac{f_{s}}{N}\right),
$$

where $f_{s}$ is the sampling frequency, and $W_{R}(f)$, known as the aliased sinc (asinc) function, is the DTFT of a zerocentered rect function of length $N$ :

$$
W_{R}(f)=\mathcal{F}\{\Pi[n]\}=\frac{\sin (\pi f N)}{\sin (\pi f)} .
$$

In Eq. (5), $\mathcal{F}($.$) is the DTFT operator. Thus, W_{H}(f)$, shown in Fig. 6(a) (solid blue trace), is a sum of three weighted and frequency-shifted as inc functions (dashed traces). A spectral footprint of the Hamming window is inversely proportional to the number of taps, with the first null occurring at $f_{s} / N=$ $32 \times 10^{9} / 201=318.4 \mathrm{MHz}$.

Frequency response of the resulting low-pass filter, depicted in Fig. 6(b), is given by a convolution of $W_{H}(f)$ with an ideal rectangular window $\Pi(f)$ (solid green trace in Fig. 6(a)):

$$
H_{\mathrm{LPF}}(f)=W_{H}(f) * \Pi(f)
$$

Since our desired cutoff frequency is $20 \mathrm{MHz}$ (see [17]), which is considerably lower than the Hamming window's main lobe width, the result of Eq. (6) is a slight spectral spread of the main lobe, so that the first null shifts from 318.4 to 324.2 $\mathrm{MHz}$, and the 3-dB cutoff frequency is $104.2 \mathrm{MHz}$.
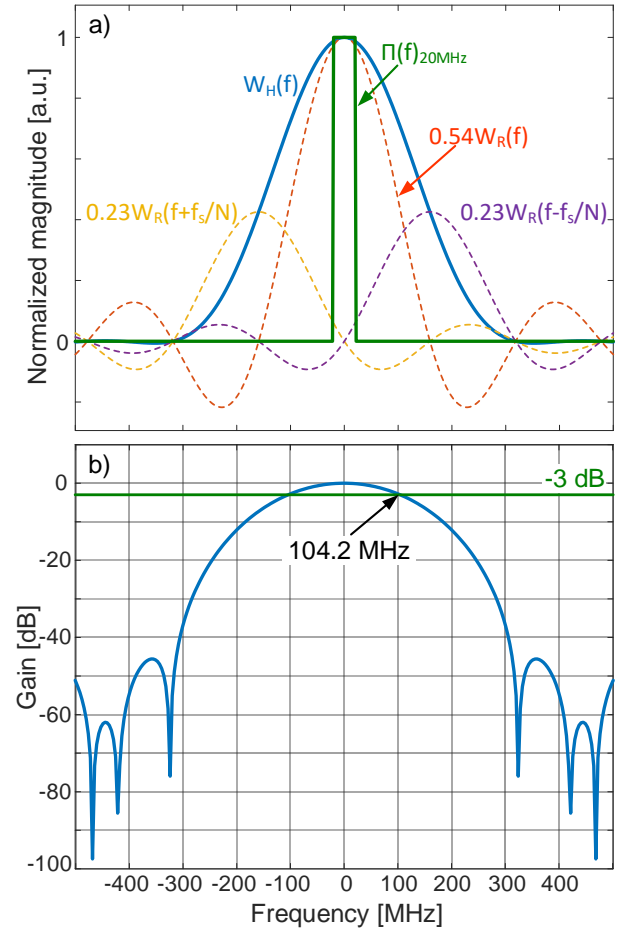

Fig. 6. F-CPE low-pass filter: a) frequency-domain Hamming window representation; (b) magnitude response of the resulting filter. $f_{s}-$ sampling frequency $=32 \mathrm{GSa} / \mathrm{s}$ (same as the symbol rate); $N$ - number of filter taps $=201$.

\section{B. FFT size optimization for low-pass filtering}

As aforementioned, one advantage of F-CPE is that lowpass filtering can be efficiently implemented in the frequencydomain using FFT, whose size can be optimized to reduce power consumption. In the following, we use the methodology presented in [24] to find the optimal FFT size. We assume a radix-2 Cooley-Tukey algorithm, and a standard complex multiplication implementation by four real multiplications and two real additions. Under these conditions, the number of nontrivial $^{1}$ real multiplications and real additions for each FFT computation is given by [25]:

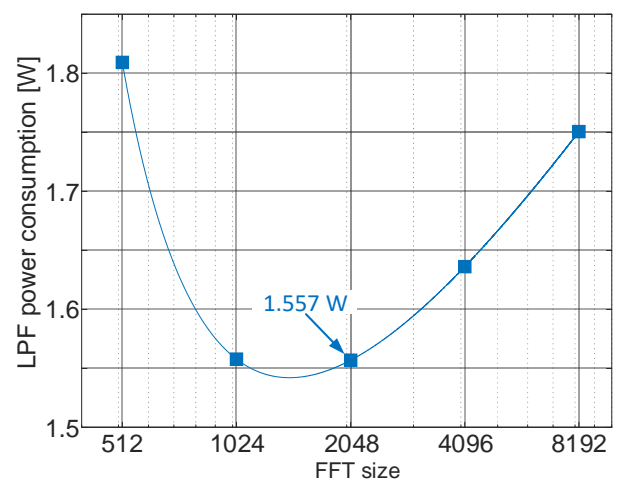

Fig. 7. Power consumption of the low-pass filter as a function of FFT size.

\footnotetext{
${ }^{1}$ In this context, a trivial multiplication is defined as a multiplication by $(-1)^{k}$ for $k=0,1$ [25].
} 

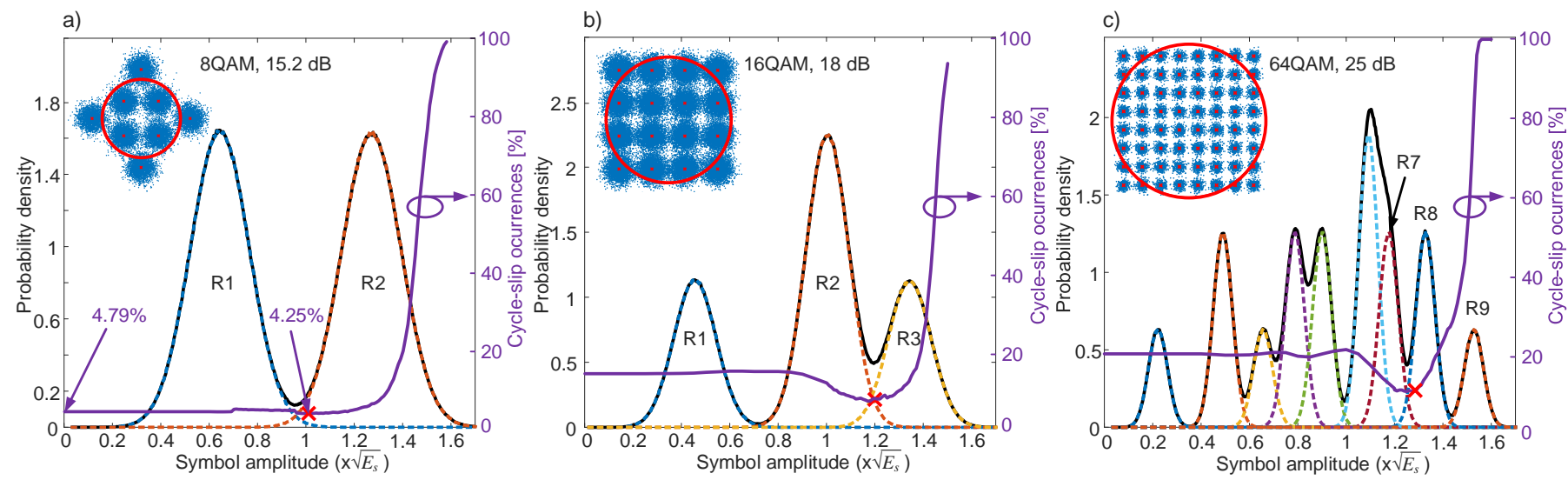

Fig. 8. Probability density functions of symbol magnitude distribution, and the cycle-slip occurrences for: (a) 8QAM; (b) 16QAM; and (c) 64QAM. Solid black traces - distribution density for all symbols; dashed colored traces - distribution densities for individual rings; Solid purple traces (right-hand-side axes) - cycle-slip occurrences, averaged over all SNR values. Red crosses represent the chosen threshold values. Insets: optimal QPSK partition threshold positions (red circles) for the respective constellations.

$$
\begin{aligned}
M_{R} & =2 N_{\mathrm{FFT}}\left(-3+\log _{2} N_{\mathrm{FFT}}+8\right) \text { and } \\
A_{R} & =3 N_{\mathrm{FFT}}\left(-1+\log _{2} N_{\mathrm{FFT}}+4\right),
\end{aligned}
$$

respectively, where $N_{\mathrm{FFT}}$ is the FFT size, such that $N_{\mathrm{FFT}}=$ $2^{k}, k \in \mathbb{N}$.

Each filtering cycle contains (i) FFT computation of a new-coming data-block; (ii) its term-by-term multiplication by FFT of the LPF coefficients; and (iii) computation of the inverse FFT (IFFT) of the result. When using long sequence filtering methods, such as overlap-\&-save, or overlap-\&-add, each filtering cycle produces $N_{\mathrm{FFT}}-N_{\mathrm{LPF}}+1$ aliasing-free symbols, where $N_{\mathrm{LPF}}$ is the number of filter taps. Therefore, the number of real multiplications and additions per filtered symbol is given by:

$$
\begin{aligned}
M_{s} & =\frac{2 M_{R}+4 N_{\mathrm{FFT}}}{N_{\mathrm{FFT}}-N_{\mathrm{LPF}}+1} ; \\
A_{s} & =\frac{2 A_{R}+2 N_{\mathrm{FFT}}}{N_{\mathrm{FFT}}-N_{\mathrm{LPF}}+1} .
\end{aligned}
$$

In Eqs. (9-10), factor 2 that multiplies $M_{R}$ and $A_{R}$ accounts for both the FFT and the IFFT, and the factors 4 and 2 that multiply $N_{\mathrm{FFT}}$ correspond to real multiplications and real additions per complex multiplication, respectively.

We next use the energy consumption approximation for an $N_{b}$-bit real multiplier and $N_{b}$-bit real adder operations, proposed in [26]:

$$
\begin{aligned}
E_{m} & =2.57 N_{b}^{2} P_{\mathrm{CMOS}} V_{c c}^{2}[\mathrm{fJ}] ; \\
E_{a} & =2.57 N_{b} P_{\mathrm{CMOS}} V_{c c}^{2}[\mathrm{fJ}],
\end{aligned}
$$

where $P_{\text {CMOS }}$ is the CMOS process technology (in $\mathrm{nm}$ ), and $V_{c c}$ is the supply voltage. Finally, LPF power consumption is given by:

$$
P=\left(E_{m} M_{s}+E_{a} A_{s}\right) \times R_{s},
$$

where $R_{s}=1 / T_{s}$ is the symbol rate.

Fig 7 shows the low-pass filtering power consumption as a function of FFT size using the following parameters: $N_{b}=6$; $P_{\mathrm{CMOS}}=16 \mathrm{~nm} ; V_{c c}=0.8 \mathrm{~V} ; N_{\mathrm{LPF}}=201$ taps; and $R_{s}=32$ GBd. Under these conditions, two optimal $N_{\text {FFT }}$ values are
1024 and 2048, corresponding to power consumptions of 1.558 $\mathrm{W}$ and $1.557 \mathrm{~W}$, and overlaps of $\left(N_{\mathrm{LPF}}-1\right) / N_{\mathrm{FFT}}=19.5 \%$ and $9.8 \%$, respectively.

\section{F-CPE for modulation formats with outer-ring QPSK sym- metry}

We begin our investigation by finding optimal thresholds for the QPSK partition of 8, 16, and 64QAM constellations. To that end, we set the laser linewidth to $1.5 \mathrm{MHz}$ (an arbitrary value with a non-negligible probability of cycle-slip occurrence for all tested SNRs) and swept through threshold and SNR values, while counting the number of trials that presented cycle slips. The SNR values, summarized in Table I, were chosen to cover BER values compatible with the hard and soft-decision FEC codes, approximately between $10^{-4}$ and $3 \times 10^{-2}$. Figs. $8(\mathrm{a}-\mathrm{c})$ show probability density functions of symbol magnitude distribution for 8,16, and 64QAM, for arbitrary SNRs of $15.2,18$, and $25 \mathrm{~dB}$, respectively, chosen for better visualization.

TABLE I

SNR SWEEPS FOR THRESHOLD ANALYSIS

\begin{tabular}{r|ccc} 
& \multicolumn{3}{|c}{ SNR range [dB] } \\
& min & step & max \\
\hline 8QAM & 10.0 & 0.4 & 15.2 \\
16QAM & 12.4 & 0.4 & 18.0 \\
64QAM & 18.2 & 0.4 & 30.0
\end{tabular}

For each pair of threshold and SNR, we conducted 200 independent trials with $10^{6}$ symbols each. The solid purple lines in Figs. 8(a-c), referred to the right-hand-side axes, show the average percentage of cycle-slip occurrence. A trial was counted as presenting cycle slips if the phase error exceeded $\pm 85^{\circ}$ for any symbol. Here, the number of cycle slips was averaged over all SNR values. For example, each point on the purple trace for 8QAM (Fig. 8(a)) was averaged over $200 \times$ $14=2800$ independent trials ( 14 being the number of tested SNR values). In this way, $10 \%$ cycle slips means that in 280 

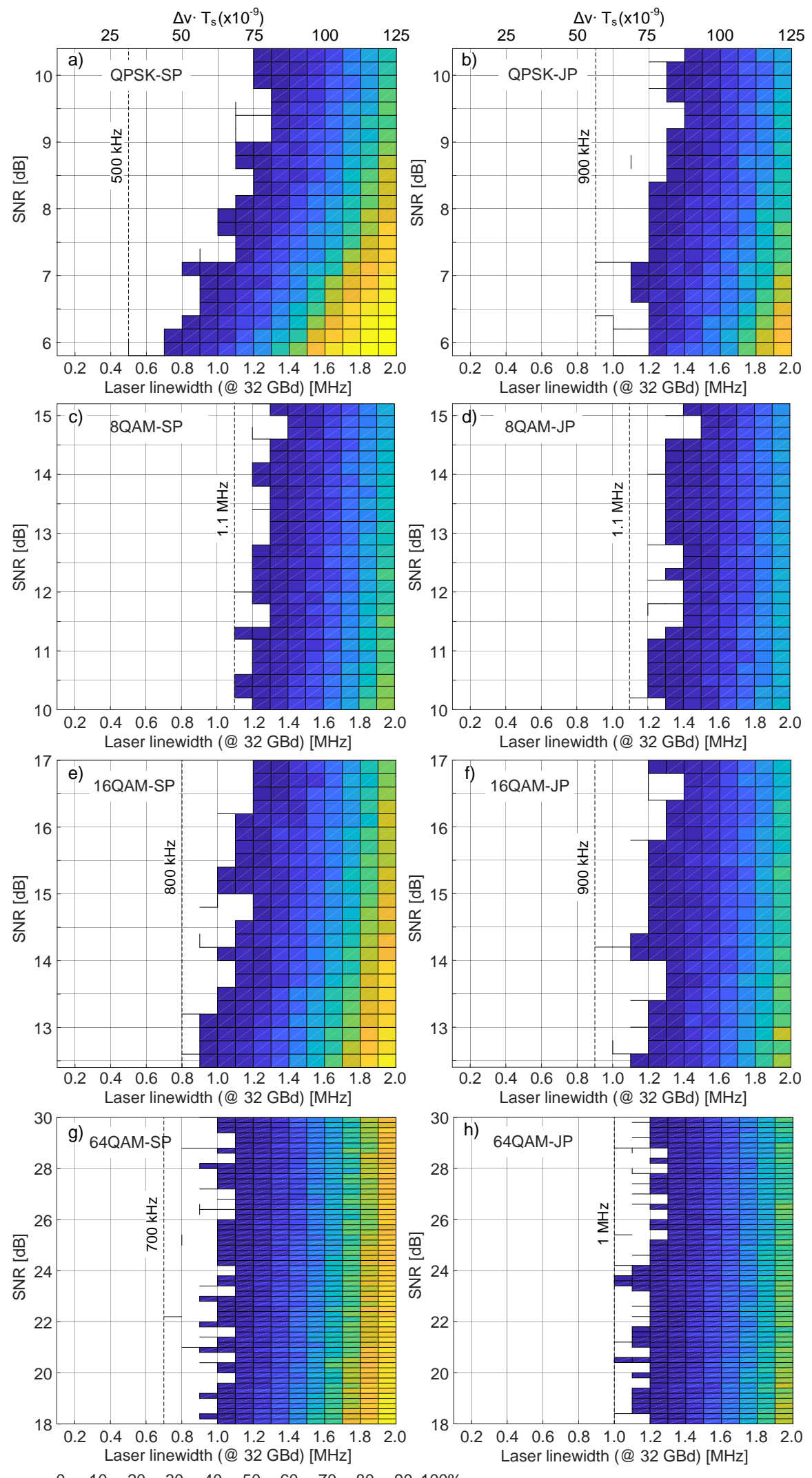

$\begin{array}{llllllllll}10 & 20 & 30 & 40 & 50 & 60 & 70 & 80 & 90 & 100 \%\end{array}$

Fig. 9. Percentage of cycle-slip occurrences as a function of SNR and laser linewidth at symbol rate 32 GBd. Dashed vertical lines show the position of first cycle-slip occurrences. 

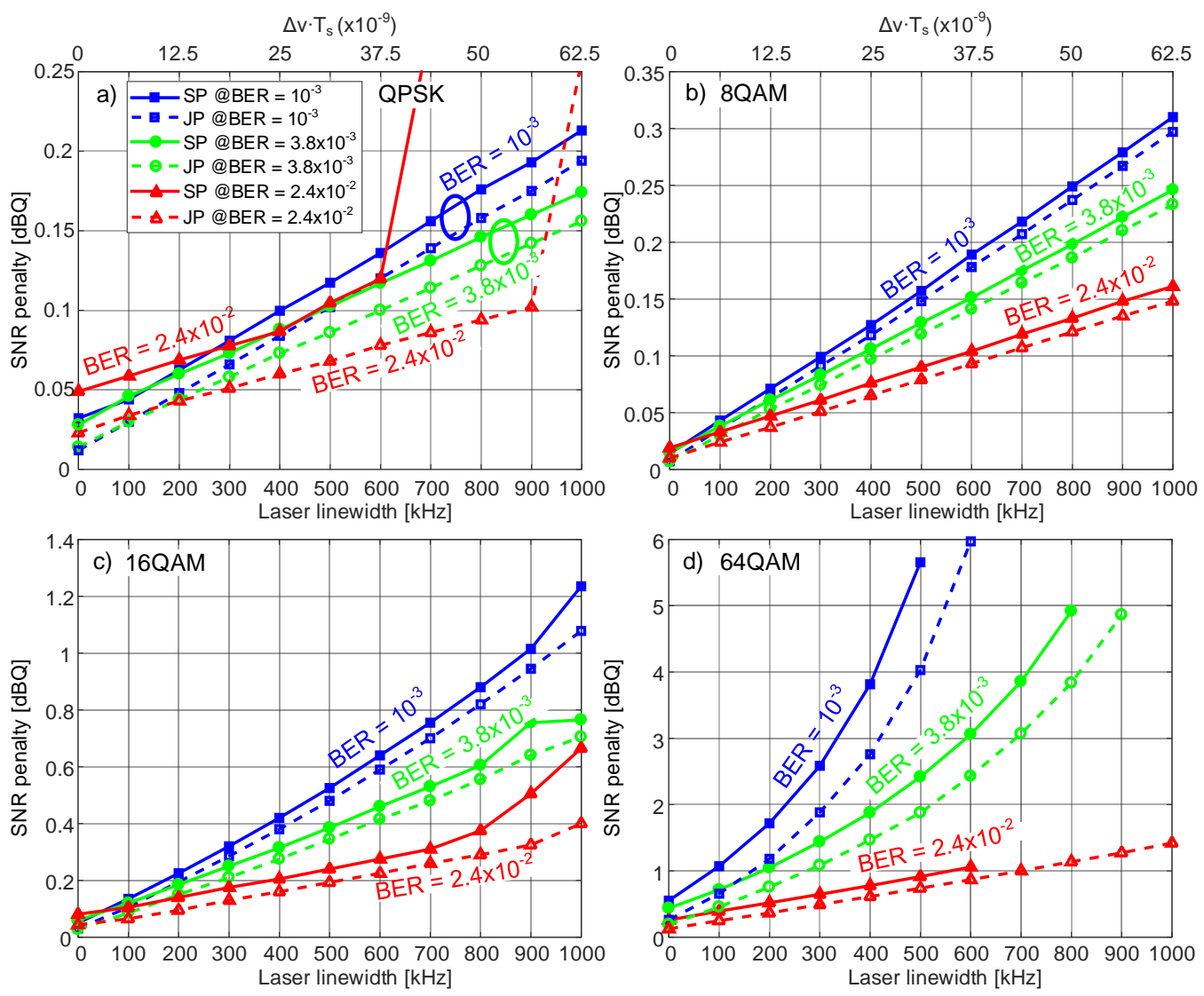

Fig. 10. F-CPE SNR penalty as a function of laser linewidth for selected BER values.

out of 2800 trials phase error magnitude exceeded $85^{\circ}$ for at least one symbol.

For 8 and 16QAM, the optimal QPSK partition threshold values, in the sense of minimum cycle-slip occurrences (marked with red crosses in Fig. 8), lie near the decision threshold, i.e., near the crossing of individual probability density functions (dashed colored traces) of the two outer rings, R1-R2 for 8QAM, and R2-R3 for 16QAM. Note that for 16QAM (Fig. 8(b)) this decision threshold depends on SNR, since R2 and R3 are not equiprobable. For 8QAM (Fig. 8(a)), the minimum cycle-slip occurrence of $4.25 \%$ corresponds to the threshold value $1.01 \sqrt{E_{s}}$, which is only marginally lower (less that 1\%) than cycle-slip occurrence at threshold zero $(4.79 \%)$, suggesting that QPSK partition can, in fact, be dropped with only a small penalty.

For 64QAM (Fig. 8(c)), the optimal threshold occurs at $1.28 \sqrt{E_{s}}$, slightly above the R7-R8 decision threshold, so that the QPSK partition includes symbols from the second outmost ring, R8. Including the R8-symbols into QPSK partition of 64QAM was previously proposed in [7], where R8\&R9 symbols were referred to as the triangle edge. Our results corroborate the expediency of this approach.

In the rest of this work we set the thresholds to $1.01 \sqrt{E_{s}}, 1.2 \sqrt{E_{s}}$, and $1.28 \sqrt{E_{s}}$, for 8, 16 and 64QAM, as indicated in Fig. 8 by red crosses. The three constellations with the corresponding thresholds are shown in the insets. The outcomes in Fig. 8 suggest that F-CPE is tolerant to errors in outer ring selection, which is different to the strategy in [3], where the authors set their thresholds for 16QAM precisely at $r_{1}=\sqrt{E_{s} / 5}$ and $r_{3}=\sqrt{9 E_{s} / 5}$, to minimize decision errors (cf. Fig. 2 of [3]).

Fig. 9 shows the percentage of occurrences of cycle slips, as a function of laser linewidth and SNR. Each pair of figures in the same row corresponds to the same modulation format, and the columns correspond to either single-polarization processing (SP, left column), or joint-polarization processing (JP, right column). For each pair of tested linewidth \& SNR values, 200 independent trials were conducted with $10^{6}$ symbols each. As previously, a trial was counted as presenting cycle slips if the phase error exceeded $\pm 85^{\circ}$ for any symbol. White spaces in the figure correspond to regions where no cycle slips were observed. For reference, the figure also presents symbol-rateindependent axes of the corresponding $\Delta \nu T_{s}$ values (top). Note that the bottom axes refer to a single laser linewidth, while in the upper axes $\Delta \nu T_{s}$ refers to the aggregate linewidth of transmitter and local oscillator lasers. Dashed vertical lines show the position of first cycle-slip occurrences.

For joint-polarization processing, at $32 \mathrm{GBd}$, first cycle slips appear at $900-\mathrm{kHz}$ laser linewidth $(1.8 \mathrm{MHz}$ aggregate linewidth) for QPSK and 16QAM, and at $\geq 1 \mathrm{MHz}$ for 8 and 64QAM, outperforming SP in all cases. For the singlepolarization processing, the first cycle slips appear at 500, 1100,800 and $700 \mathrm{kHz}$ for QPSK, 8, 16 and 64QAM, respectively. These results make F-CPE attractive for flexible transceivers that support different modulation formats and use high quality lasers, such as ECL, whose linewidth is in the 


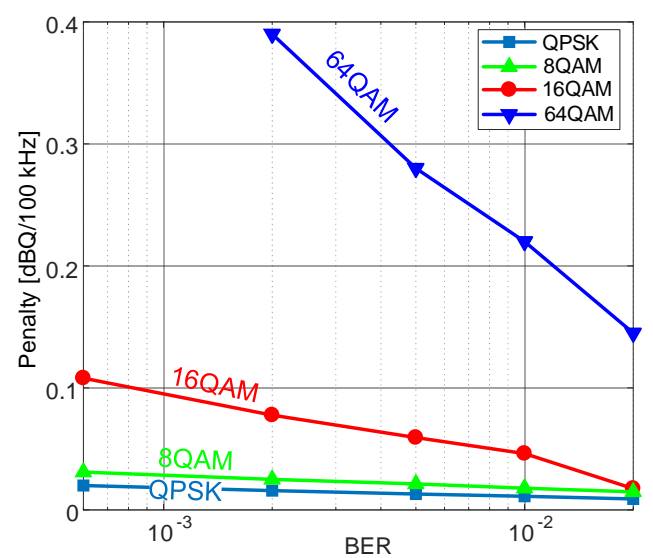

Fig. 11. Change in SNR penalty as a function of BER, extrapolated from BER vs. SNR curves for laser linewidths below cycle-slip thresholds.

range of up to a few hundred $\mathrm{kHz}$.

With the exception of QPSK, cycle-slip occurrence is only weakly dependent on SNR, as targeted by the aggressive additive noise rejection strategy of F-CPE. Conversely, for QPSK there is a clear dependency of number of cycle slips on signal SNR, especially in the single-polarization processing case. This is likely because for QPSK there is no noise rejection through QPSK partition. Also, for similar BER values, QPSK operates at a much higher noise load than the other investigated modulation formats.

We next assess the sensitivity penalties induced by FCPE. Fig. 10 shows SNR penalties in comparison with the AWGN-only scenario (without applying carrier recovery), as a function of laser linewidth for three selected BER values: $10^{-3}, 3.8 \times 10^{-3}$, and $2.4 \times 10^{-2}$. Every point on the traces is an average of 200 individual trials. Jumps in some of the traces in the high-linewidth region are due to cycle slips. With the exception of 64QAM, SNR penalties increase exponentially (linearly in $\mathrm{dB}$ ), and this increase is identical for single- and joint-polarization processing. The rate of penalty increase is different for different BER values, and is the smallest for low signal quality, where phase noise penalty is masked by the additive noise. Further, joint-polarization processing shows a slight performance improvement in comparison with the single polarization processing, though, these differences are probably too small to impact system design process. For BER $=10^{-3}$ and $3.8 \times 10^{-3}, 64$ QAM shows rapid penalty growth with laser linewidth due to a performance floor, experienced by the BER. For linewidth $=100 \mathrm{kHz}$, comparable with modern ECL lasers, the joint-polarization processing penalties are $\leq 0.05$ $\mathrm{dB}$ for QPSK and 8QAM, $\leq 0.1 \mathrm{~dB}$ for 16QAM, and $\leq 0.65$ $\mathrm{dB}$ for 64QAM. These penalties can be seamlessly included within the system margin.

Lastly, Fig. 11 shows the change in SNR penalty as a function of BER. That is, it shows the changes in slope of the traces in Fig. 10. Thus, for 16QAM at BER $=10^{-3}$, an increase of $100 \mathrm{kHz}$ in laser linewidth yields additional $\sim 0.1$ $\mathrm{dB}$ SNR penalty, while at $\mathrm{BER}=10^{-2}$, additional penalty is $\sim 0.05 \mathrm{~dB}$. For QPSK and 8QAM, additional penalty for a $100-\mathrm{kHz}$ linewidth increase is below $0.03 \mathrm{~dB}$ for BER $\geq 10^{-3}$.
64QAM shows the highest penalties, however, it is expected to operate at high BER values, where the incremental penalty is at its minimum. The information in Fig. 11 was extrapolated from BER vs. SNR curves for laser linewidths below cycle-slip thresholds in Fig. 9. For 64QAM, the points for BER $2 \times 10^{-3}$ and $5 \times 10^{-3}$ were averaged over laser linewidths up to 400 $\mathrm{kHz}$, because of the nonlinear behavior (in $\mathrm{dB}$ ) of the penalty curves in this region (Fig. 10(c)).

\section{Comparison of F-CPE with BPS and data-aided phase estimators}

Next, we compare the performance of F-CPE with that of a blind phase search algorithm (BPS) and a data-aided phase estimator, in terms of BER, phase noise resilience, and cycleslip tolerance.

There are three parameters that affect BPS performance: $(i)$ the number of test phases, $B$; (ii) size of the noise rejection window, $N$; and (iii) the step-size, $s$. The parameter $B$ sets phase search granularity, and, in principle, should increase with the modulation order. The parameter $N$ is responsible for additive noise rejection. If increased excessively, it may lead to performance penalties due to reduced phase noise correlation. The step-size $s$ defines the periodicity of phase computation. Increasing $s$ reduces the computational burden on BPS, by taking advantage of slowly varying nature of the phase noise. The computational complexity of BPS is approximately proportional to $B N / s$. In the following comparison, we use a practical case BPS configuration: $B=20$ (yielding a granularity of $90^{\circ} / 20=4.5^{\circ}$ ), $N=20$, and $s=10$, further referred to as BPS 20/20/10, which provides a reasonable trade-off between the computational complexity and performance.

There are many possibilities for implementing a data-aided phase estimator. In principle, any blind estimator can be extended to benefit from the information obtained from pilot symbols, as, e.g., in [27], where the authors extended the algorithm of [28] to avoid cycle slips. In this work, we use a naïve data-aided phase estimator, described in Fig. 12. Let the received symbol train be composed of data blocks $D$ of length $L_{D}$, interleaved with pilot symbol blocks $P$ of length $L_{P}$. Let $a_{1} \ldots a_{L_{P}}$ and $x_{1} \ldots x_{L_{P}}$ be the sent and the received pilot symbols of an $n$-th pilot block, respectively. Then, the phase error for this pilot block is calculated as the average value [27]:

$$
\theta_{n}^{P}=\frac{1}{L_{P}} \sum_{k=1}^{L_{P}} P U_{1}\left\{\arg \left(a_{k}^{*} \cdot x_{k}\right)\right\} .
$$

The phase unwrapping operation $P U_{1}$ within the pilot block is required for the arg defined in the interval $(-\pi, \pi]$ when phase values within one $P$ block oscillate around $\pi$, changing their sign (alternatively, around zero, if arg is defined in [0, $2 \pi)$ ), to result in a meaningful mean value ${ }^{2}$. Observe that $P U_{1}$ is different from $\mathrm{PU}_{4}$ in Eq. (1), which unwraps the phase

\footnotetext{
${ }^{2}$ To illustrate that, assume two points on the unit circle with phases $\pi \pm \delta$. The correct average of the phases is $(\pi-\delta+\pi+\delta) / 2=\pi$. However, when the phases are defined in $(-\pi, \pi], \pi+\delta=-\pi+\delta$, so that $(\pi-\delta-\pi+\delta) / 2=0$, which is false.
} 


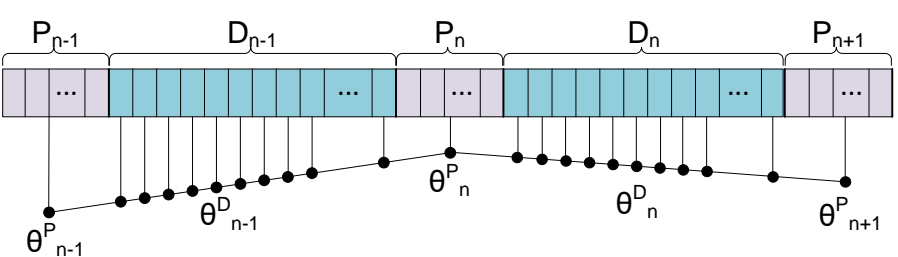

Fig. 12. Implementation of the data-aided phase estimator.

from within a $(-\pi / 4, \pi / 4]$ interval. Finally, phase error values $\theta_{1}^{D} \ldots \theta_{L_{D}}^{D}$ for the $n$-th data block $D$ are found using linear interpolation:

$$
\theta_{k}^{D}=\theta_{n}^{P}+\frac{\theta_{n+1}^{P}-\theta_{n}^{P}}{L_{D}} k
$$

where $\theta_{n}^{P}$ and $\theta_{n+1}^{P}$ are mutually unwrapped.

In this work, we set the overhead for pilot symbols to 4 percent; however, the algorithm performance is also affected by the way those symbols are distributed within data. Possible examples of $4 \%$-overhead schemes are: $\left\{L_{P}=1, L_{D}=24\right\}$; $\left\{L_{P}=20, L_{D}=480\right\}$, etc.

First, we find the optimal pilot symbol distribution by changing the length of the pilot blocks. Fig. 13 shows the average BER as a function of the pilot block length for a 16QAM constellation, where SNR is varied between 12.4 and $18 \mathrm{~dB}$ in steps of $0.4 \mathrm{~dB}$, and laser linewidth is set to 1 $\mathrm{MHz}$. On average, BER is maximized when pilot distribution is $\left\{L_{P}=4, L_{D}=96\right\}$, however, the optimal $L_{P}$ values oscillate between 3 and 6 symbols, depending on SNR (not shown in the figure). Thus, at a low SNR of $12 \sim 13 \mathrm{~dB}$, $L_{P \text { optimal }}=6$, whereas at SNR $=16 \sim 18 \mathrm{~dB}$, the optimal $L_{P \text { optimal }}=3$. In the following, we set pilot distribution to $\left\{L_{P}=4, L_{D}=96\right\}$. Here, we disregard the practicality of such a scheme in terms of its accommodation within the OTN frames, considering only the physical layer communication.

For a fair comparison, the pilot block overhead penalty is taken into account. Assuming identical optical SNR (OSNR) for blind and data-aided schemes, and using the relationship $\mathrm{SNR} \propto T_{s}$ OSNR [29], for a $4 \%$ overhead, the SNR penalty is $10 \log _{10} 1.04=0.17 \mathrm{~dB}$. On the other hand, reduced symbol time $T_{s}$ also reduces the phase noise variance by a factor of 1.04 .

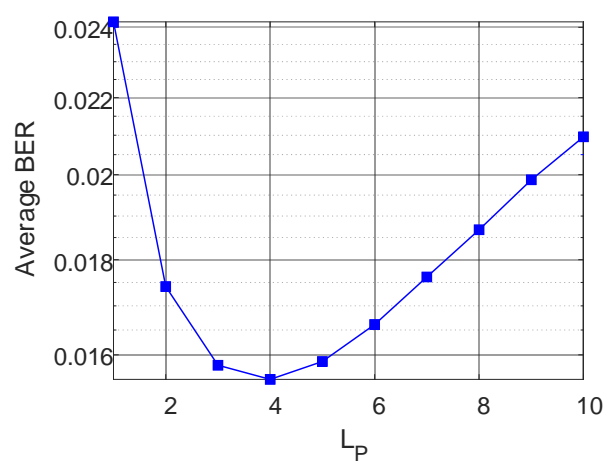

Fig. 13. Average BER versus length of symbol blocks, $L_{p}$, for a 16QAM modulation, for SNR between 12.4 and $18 \mathrm{~dB}$ and laser linewidth $1 \mathrm{MHz}$.

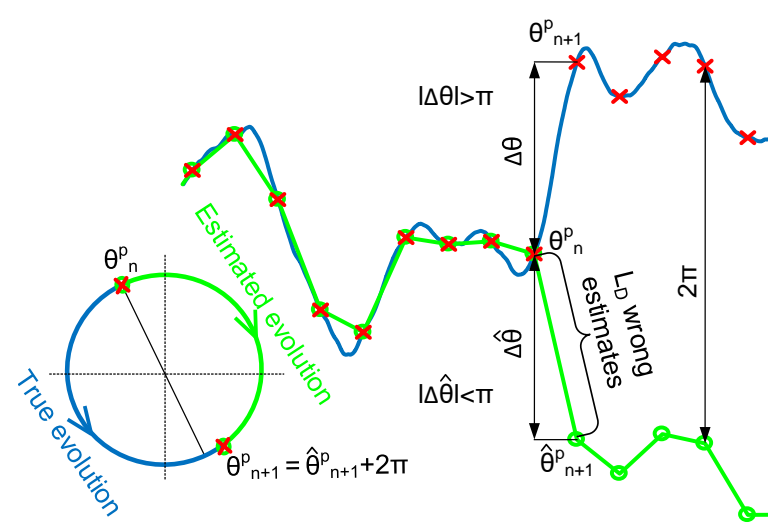

Fig. 14. Cycle-slip mechanism of a data-aided estimator. Blue - true phase evolution; green - estimated phase evolution; red crosses - phase values at pilot positions. Inset: phase evolution in the phase-quadrature plane.

At this point we would like to clarify what we consider to be a cycle slip of a data-aided estimator, since, unlike blind phase estimators, it is not insensitive to constellation rotations by $\pi / 2$. Such a cycle-slip occurrence is illustrated in Fig. 14. The figure shows the true (blue) and the estimated (light green) phases of a signal, with phase values at pilot positions indicated by red crosses. If the absolute difference between two consecutive phase values estimated from pilot sequences is greater than $\pi$ (that is, if $|\Delta \theta|=\left|\theta_{n+1}^{P}-\theta_{n}^{P}\right|>\pi$ ), the phase unwrapper $P U_{1}$ is triggered, adding $\pm \pi$, so that the estimated phase jump is smaller than $\pi:|\Delta \hat{\theta}|=\left|\hat{\theta}_{n+1}^{P}-\theta_{n}^{P}\right|<\pi$. In this way, $\left|\hat{\theta}_{n+1}^{P}-\theta_{n+1}^{P}\right|=2 \pi$. Naturally, the phase is indifferent to $2 \pi$-jumps, however its evolution between the $n$ th and the $(n+1)$-th pilot blocks is wrongly estimated, causing the interpolator in Eq. 15 to produce catastrophic errors for $L_{D}$ symbols of the $n$-th data block. The inset illustrates this concept in the phase-quadrature plane. Clearly, the likelihood of such slips increases with $L_{D}$.

Fig. 15 shows the BER as a function of laser linewidth for the three tested phase estimators, where each pair of figures in the same row corresponds to the same modulation format. Figures in the left column (Figs. 12 (a,c,e,g)) correspond to the BER obtained with differential decoding, which show the estimators' sensitivities without the impact of cycle slips; and the figures in the right column (Figs. 12 (b,d,f,h)) correspond to Gray decoding, so that cycle slips are manifested as jumps in the BER curves. Each figure has three sets of curves, shown in different line styles (solid, dashed, and dashed-dotted), obtained for three different SNR values (provided in the legend). The figures also show AWGN-only BER thresholds (black unmarked traces). The SNR values were chosen to cover the range of pre-FEC BERs, compatible with modern hardand soft-decision error correction codes, used in metro and long-haul optical transmission systems.

The results for differential decoding (left column of Fig. 15) show that the a priori sensitivities (that is, without the impact of cycle slips) of F-CPE and BPS are similar for QPSK and 8QAM, where BPS has a slightly smaller linewidth increase penalty at high SNRs (dashed-dotted traces). For 16 and 64QAM, BPS is more robust to an increase in laser 

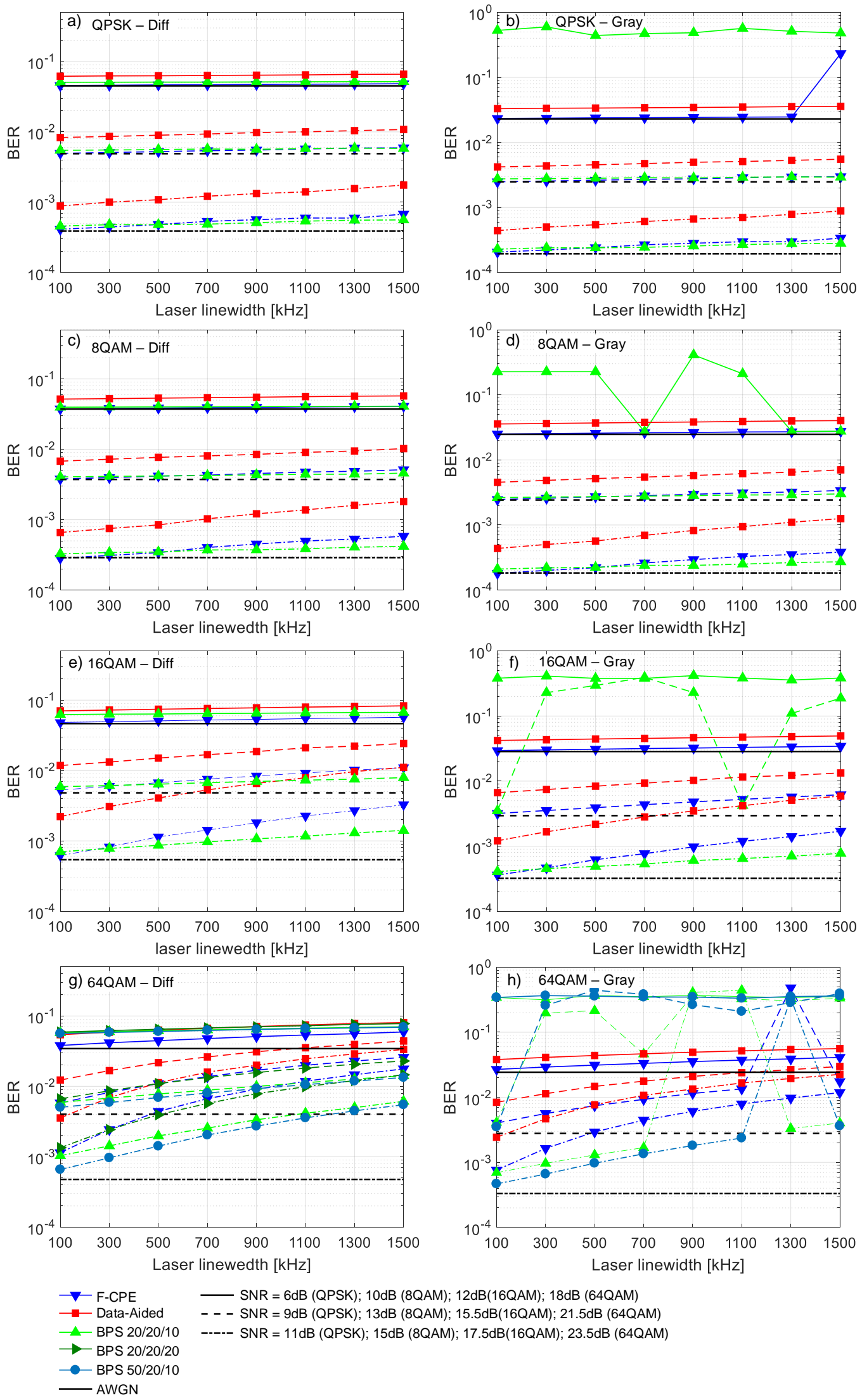

Fig. 15. BER vs. laser linewidth for F-CPE, BPS, and a data-aided phase estimator. Left column (a,c,e,g): differential decoding; right column (b,d,f,h): Gray decoding. 
linewidth, outperforming F-CPE at high SNRs for $\Delta \nu \geq 300$ $\mathrm{kHz}$. On the other hand, F-CPE shows greater robustness against additive noise, outperforming the BPS at low SNRs, as expected from [17]. The traces for a $4 \%$ data-aided estimator follow the same pattern as F-CPE, indicating identical laser linewidth penalties, with an inferior overall sensitivity. To emphasize the dependency of BPS on the chosen parameters, Fig. $15(\mathrm{~g})$ also shows the performance of BPS with step-size $s=20$ (BPS 20/20/20) for 64QAM. Under this configuration, the sensitivity of BPS is quasi-identical to F-CPE, even in a high SNR regime.

The results obtained with Gray decoding show that BPS is completely overtaken by cycle slips at low SNR, compatible with the soft-decision FEC schemes (solid traces), producing constant $\mathrm{BER} \approx 0.5$ for most modulation formats. At high SNR, BPS shows a better cycle-slip robustness for QSPK and 8QAM; however, it presents cycle slips for high-order modulation formats: at SNR $=15.5 \mathrm{~dB}$ for 16QAM, and at $\mathrm{SNR}=\{17.5,23.5\} \mathrm{dB}$ for 64QAM. F-CPE showed two occurrences of cycle slips, for QPSK at $\Delta \nu=1.5 \mathrm{MHz}$, and for 64QAM at $\Delta \nu=1.3 \mathrm{MHz}$. Both cases statistically agree with the outcomes of Fig. 9. As expected, the dataaided algorithm showed a superior cycle-slip robustness, by not presenting any cycle slips throughout the tested conditions. This is because, as earlier mentioned, the data-aided estimator does not suffer from a $90^{\circ}$ phase ambiguity. Additionally, a relatively small length $L_{D}$ in the $\left\{L_{P}=4, L_{D}=96\right\}$ scheme guarantees a high phase correlation within data blocks, $D$, making it statistically unlikely for the phase to evolve differently from the predictions of Eq. (15).

For 64QAM, the phase resolution of BPS of $90^{\circ} / 20=4.5^{\circ}$ is generally too low, and can increase performance penalties. Therefore, in Figs. 15(g,h), we include the performance of BPS $50 / 20 / 10$ (phase granularity $90^{\circ} / 50=1.8^{\circ}$ ). Indeed, increasing phase resolution results in better sensitivity for high SNRs and low laser linewidths (see Fig. 15(g)); however, at low SNRs, corresponding to BER $>10^{-2}$, this sensitivity improvement is negligible. Also, the cycle-slip robustness of BPS appears unaffected by the increased phase resolution (Fig. 15(h)).

\section{E. F-CPE for $32 Q A M$}

Under certain transmission conditions, 32QAM can exhibit benefits over other modulation formats in terms of rate $v s$. reach trade-offs, and is commonly considered in the scope of flexible optical transceivers. Yet, 32QAM does not possess outer-ring symbols with a QPSK symmetry, which hinders the use of F-CPE. Nevertheless, as we show next, F-CPE can still be used with 32QAM, albeit, with higher sensitivity penalties and lower robustness against cycle slips.

Fig. 16 shows how F-CPE interacts with a 32QAM modulation. The 32QAM constellation, depicted in Fig. 16(a), has five radii, R1-R5, where the R5 symbols form two rotated QPSK constellations, indicated by green and red crosses. When the constellation is raised to the 4-th power, these symbols are mapped onto two points, symmetric around the quadrature axis, so that the phase of their average is $\pi$. Suppose the constellation is rotated by $\Delta \theta$, as depicted by blue circles in
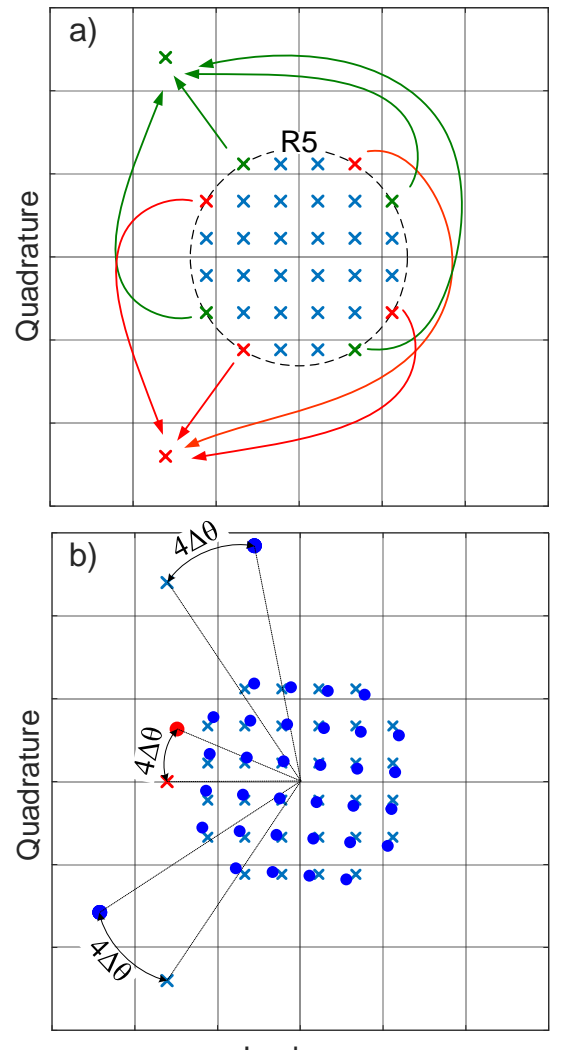

In phase

Fig. 16. F-CPE interaction with a 32QAM constellation. (a) Outer symbols R5 4-th-power mapping. (b) Rotated constellation 4-th-power mapping - phase information is preserved.

Fig. 16(b). When raised to the 4-th power, the R5 symbols are mapped onto two points that are rotated to the same direction by $4 \Delta \theta$ from the ideal 4-th power mapping points. An average of these two points (unwrapped, so that there is no sign inversion around $\pi$ ) will deviate in phase by $4 \Delta \theta$ from $\pi$, preserving, on average, the phase information.

As in Section III-C, we find the optimal QPSK-partition ${ }^{3}$ threshold value in terms of cycle-slip occurrences, using laser linewidth $\Delta \nu=1.5 \mathrm{MHz}$, sweeping through threshold and SNR values (SNR range 16 to $22 \mathrm{~dB}$ in steps of $0.4 \mathrm{~dB}$ ), and counting the trials that presented cycle slips. Fig. 17 shows that the optimal threshold value is $1.24 \sqrt{E_{s}}$, so that the QPSK partition almost uniquely uses the R5 symbols.

Figs. 18(a-b) show the percentage of cycle-slip occurrences in 32QAM as a function of laser linewidth and SNR, for single- and joint-polarization processing, respectively. As in Fig. 9, every pair of tested laser linewidth and SNR corresponds to 200 independent trials of $10^{6}$ symbols each. A comparison between Figs. 9 and 17 indicates that F-CPE's cycle-slip robustness is greatly impaired by $32 \mathrm{QAM}$, showing a much greater dependency on the additive noise.

Figs. 19(a-b) compare the performance of F-CPE, BPS 20/20/10, and a data-aided estimator for 32QAM, showing BER vs. laser linewidth curves, obtained with differential and Gray decodings, respectively. Each figure has three sets of

\footnotetext{
${ }^{3}$ We continue to use the term QPSK partition for the threshold-based symbol nulling, although, it is inaccurate in the context of 32QAM.
} 


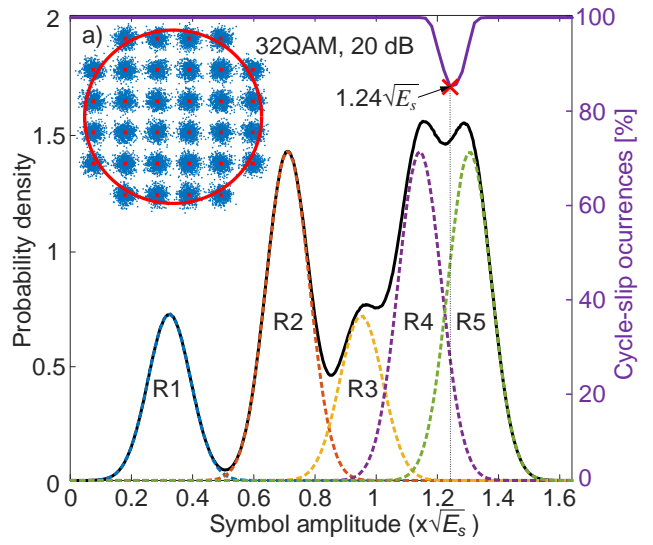

Fig. 17. Probability density functions of symbol magnitude distribution, and the cycle-slip occurrences for 32QAM. Solid black traces - distribution density for all symbols; dashed colored traces - distribution densities for individual rings; Solid purple traces (right-hand-side axes) - cycle-slip occurrences, averaged over all SNR values. Red cross represents the chosen threshold value of $1.24 \sqrt{E_{s}}$. Inset: optimal QPSK partition threshold position (red circle).
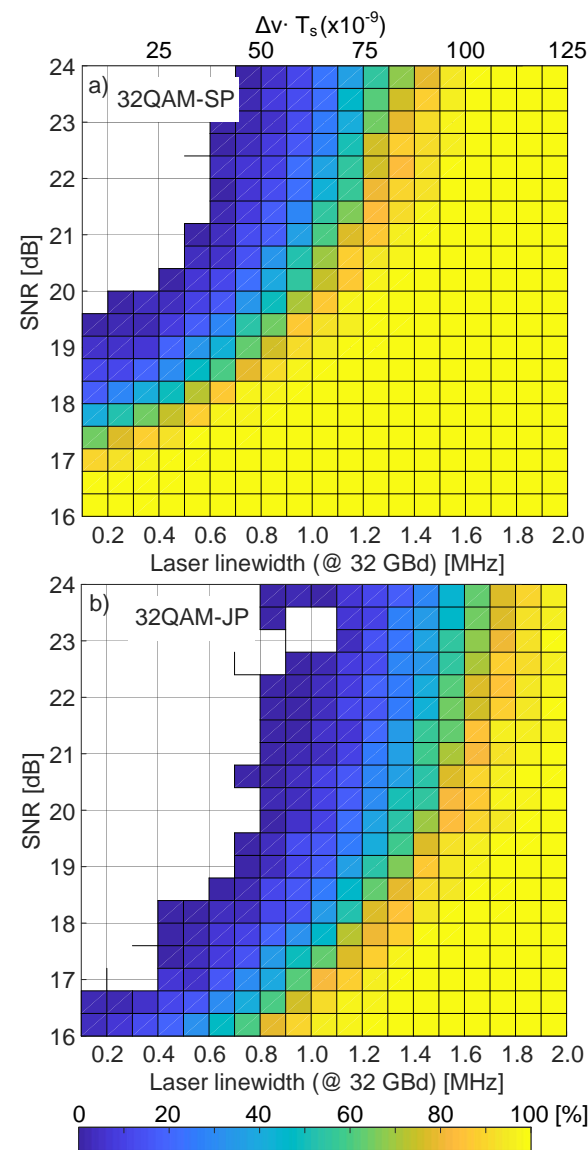

Fig. 18. Percentage of cycle-slip occurrences in 32QAM as a function of SNR and laser linewidth, at symbol rate $32 \mathrm{GBd}$.

curves, shown in solid, dashed, and dashed-dotted, obtained for three different SNR values: 16,19 , and $21 \mathrm{~dB}$, to cover the typical threshold values of modern soft and hard-decision FEC schemes. The AWGN-only BER thresholds are shown by the black unmarked traces.

The first conclusion of Fig. 19(a) is that F-CPE is able to
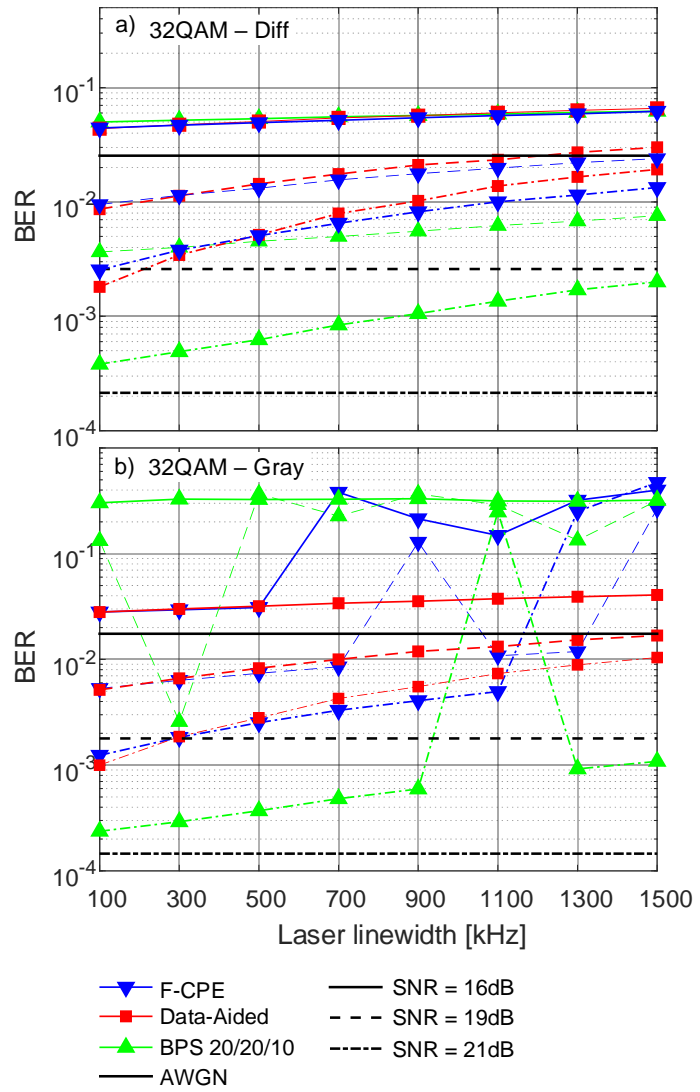

Fig. 19. 32QAM - BER vs. laser linewidth for F-CPE, BPS, and a data-aided phase estimator using (a) differential decoding; (b) Gray decoding.

track the phase noise for 32QAM even without the outerring QPSK symmetry, showing valid bit error rates for the tested range of SNR and laser linewidth values. This result validates the above discussion on F-CPE and 32QAM interaction. Secondly, BPS completely outperforms F-CPE for high SNR regime, having almost a full order of magnitude BER difference. Conversely, in a low SNR regime, both algorithms show a similar performance, with F-CPE performing slightly better for low $\Delta \nu$ values (for example, at $\Delta \nu=100 \mathrm{kHz}$, $\mathrm{BER}_{\mathrm{BPS}}=5.0 \times 10^{-2} ; \mathrm{BER}_{\mathrm{F}-\mathrm{CPE}}=4.4 \times 10^{-2}$ ). The dataaided estimator and F-CPE have similar sensitivities, where FCPE is slightly less penalized by an increase in laser linewidth.

Finally, from Fig. 19(b) it follows that F-CPE loses (at least partially) the advantage of robustness against cycle slips over BPS for 32QAM. Still, its use over BPS might be justified owing to its computational complexity benefits, if there is a sufficient signal quality margin in the system.

\section{CONCLUSION}

We have performed detailed numerical simulations of the recently proposed filtered carrier phase estimation algorithm, F-CPE, extending it to modulation formats whose outerring symbols form a QPSK constellation: QPSK, 8, 16, and 64QAM. Additionally, we have proposed a joint-polarization processing architecture that minimizes incremental complexity, and an I-Q alignment architecture that minimizes incremental footprint. Joint-polarization processing F-CPE showed 
cycle-slip-free operation for laser linewidth values below 900 $\mathrm{kHz}$, making it attractive for flexible transceivers that support different modulation formats and use narrow linewidth lasers. In this scenario, low sensitivity penalties of F-CPE can be seamlessly absorbed within system SNR margin. We have further shown that F-CPE can also be employed with 32QAM signals, though, with some penalties and reduced cycle-slip robustness. Finally, a comparison with BPS showed that in terms of sensitivity F-CPE generally outperforms BPS under low SNR, while exhibiting higher laser linewidth penalties under high SNR.

\section{ACKNOWLEDGEMENTS}

This work was supported by the Australian Research Council (FL130100041, CE110001018). At UNICAMP, this work was supported by FAPESP (2015/24341-7, 2015/24517-8).

\section{REFERENCES}

[1] M. G. Taylor, "Phase estimation methods for optical coherent detection using digital signal processing," J. Lightwave Technol., vol. 27, no. 7, pp. 901-914, April 2009.

[2] A. Viterbi and A. Viterbi, "Nonlinear estimation of PSK-modulated carrier phase with application to burst digital transmission," IEEE Trans. Inf. Theory, vol. 29, no. 4, pp. 543-551, July 1983.

[3] R. Muller and D. Mello, "Phase-offset estimation for joint-polarization phase-recovery in DP-16QAM systems," IEEE Photon. Technol. Lett., vol. 22, no. 20, pp. 1515-1517, Oct 2010.

[4] I. Fatadin, D. Ives, and S. J. Savory, "Laser linewidth tolerance for 16QAM coherent optical systems using QPSK partitioning," IEEE Photon. Technol. Lett., vol. 22, no. 9, pp. 631-633, May 2010.

[5] Y. Chen and X. G. Huang, "A linewidth-tolerant two-stage CPE using a new QPSK-partitioning approach and an enhanced maximum likelihood detection for 64-QAM coherent optical systems," J. Lightwave Technol., vol. 33, no. 18, pp. 3883-3889, Sept 2015.

[6] I. Fatadin, D. Ives, and S. J. Savory, "Carrier phase recovery for 16QAM using QPSK partitioning and sliding window averaging," IEEE Photon. Technol. Lett., vol. 26, no. 9, pp. 854-857, May 2014.

[7] S. M. Bilal, C. R. S. Fludger, V. Curri, and G. Bosco, "Multistage carrier phase estimation algorithms for phase noise mitigation in 64-quadrature amplitude modulation optical systems," J. Lightwave Technol., vol. 32, no. 17, pp. 2973-2980, Sept 2014.

[8] T. Pfau, S. Hoffmann, and R. Noe, "Hardware-efficient coherent digital receiver concept with feedforward carrier recovery for M-QAM constellations," J. Lightwave Technol., vol. 27, no. 8, pp. 989-999, April 2009.

[9] M. S. Llopis, M. S. Faruk, and S. J. Savory, "Improved linewidth toleran carrier phase recovery based on polar map metric estimate," in Proc. Opt. Fiber Commun. Conf., Los Angeles, CA, USA, 2017, p. Th4C.2.

[10] J. Navarro, A. Kakkar, R. Schatz, X. Pang, O. Ozolins, A. Udalcovs, S. Popov, and G. Jacobsen, "Blind phase search with angular quantization noise mitigation for efficient carrier phase recovery," Photonics, vol. 4, no. 37, 2017.

[11] V. N. Rozental, D. Kong, B. Foo, B. Corcoran, and A. J. Lowery, "Low complexity blind phase recovery algorithm with increased robustness against cycle-slips," in Proc. European Conf. Opt. Commun, Gothenburg, Sweden, Sept 2017, p. P1.SC3.51.

[12] E. Ip and J. M. Kahn, "Feedforward carrier recovery for coherent optical communications," J. Lightwave Technol., vol. 25, no. 9, pp. 2675-2692, Sept 2007.

[13] L. Schmalen, "A low-complexity LDPC coding scheme for channels with phase slips," J. Lightwave Technol., vol. 33, no. 7, pp. 1319-1325, Apr 2015.

[14] A. Bisplinghoff, S. Langenbach, E. S. Vercelli, R. Pastorelli, and T. Kupfer, "Cycle slip tolerant, differential encoding aware soft-decision FEC," in Proc. Opt. Fiber Commun. Conf., Los Angeles, CA, USA, March 2015, p. Tu3B.2.

[15] T. Koike-Akino, K. Kojima, D. S. Millar, K. Parsons, Y. Miyata, W. Matsumoto, T. Sugihara, and T. Mizuochi, "Cycle slip-mitigating turbo demodulation in LDPC-coded coherent optical communications," in Proc. Opt. Fiber Commun. Conf., San Francisco, CA, USA, March 2014, p. M3A.3.
[16] A. Bisplinghoff, S. Langenbach, and T. Kupfer, "Low-power, phase-slip tolerant, multilevel coding for M-QAM," J. Lightwave Technol., vol. 35, no. 4, pp. 1006-1014, Feb 2017.

[17] V. N. Rozental, D. Kong, B. Foo, B. Corcoran, and A. J. Lowery, "Cycleslip-less low-complexity phase recovery algorithm for coherent optical receivers," Opt. Lett., vol. 42, no. 18, pp. 3554-3557, Sept 2017.

[18] S. J. Savory, "Digital coherent optical receivers: algorithms and subsystems," IEEE J. Sel. Topics Quantum Electron., vol. 16, no. 5, pp. 2120-2126, 2010.

[19] F. A. Garcia, D. A. Mello, and H. Waldman, "Feedforward carrier recovery for polarization demultiplexed signals with unequal signal to noise ratios," Opt. Express, vol. 17, no. 10, pp. 7958-7969, May 2009.

[20] A. F. Alfredsson, R. Krishnan, and E. Agrell, "Joint-polarization phasenoise estimation and symbol detection for optical coherent receivers," $J$. Lightwave Technol., vol. 34, no. 18, pp. 4394-4405, Sept 2016.

[21] D. Souto, B.-E. Olsson, C. Larsson, and D. A. A. Mello, "Jointpolarization and joint-subchannel carrier phase estimation for 16-QAM optical systems," J. Lightwave Technol., vol. 30, no. 20, pp. 3185-3191, Oct 2012.

[22] J. Proakis and D. Manolakis, Digital Signal Processing: Principles, Algorithms, and Applications, 3rd ed. Prentice Hall, 1996.

[23] J. O. Smith, Spectral Audio Signal Processing, 2011, Accessed 05 April 2018. [Online]. Available: http://ccrma.stanford. edu/ jos/sasp/

[24] D. A. Mello, V. N. Rozental, T. C. Lima, F. C. Pereira, A. N. Barreto, M. Camera, and G. Bruno, "Adaptive optical transceivers: Concepts and challenges," J. Commun. Inf. Syst., vol. 29, no. 1, pp. 1-11, May 2014.

[25] R. Blahut, Fast Algorithms for Signal Processing. Cambridge University Press, 2010.

[26] B. S. G. Pillai, B. Sedighi, K. Guan, N. P. Anthapadmanabhan, W. Shieh, K. J. Hinton, and R. S. Tucker, "End-to-end energy modeling and analysis of long-haul coherent transmission systems," J. Lightwave Technol., vol. 32, no. 18, pp. 3093-3111, Sept 2014.

[27] H. Zhang, Y. Cai, D. G. Foursa, and A. N. Pilipetskii, "Cycle slip mitigation in POLMUX-QPSK modulation," in Proc. Opt. Fiber Commun. Conf., Los Angeles, CA, USA, March 2011, p. OMJ.7.

[28] M. Kuschnerov, D. van den Borne, K. Piyawanno, F. Hauske, C. Fludger, T. Duthel, T. Wuth, J. Geyer, C. Schulien, B. Spinnler, E. Schmidt, and B. Lankl, "Joint-polarization carrier phase estimation for XPMlimited coherent polarization-multiplexed QPSK transmission with OOK-neighbors," in Proc. European Conf. Opt. Commun, Brussels, Belgium, Sept 2008, p. Mo.4.D.2.

[29] R. J. Essiambre, G. Kramer, P. J. Winzer, G. J. Foschini, and B. Goebel, "Capacity limits of optical fiber networks," J. Lightwave Technol., vol. 28 , no. 4, pp. 662-701, Feb. 2010. 